\title{
Herd Immunity against Severe Acute Respiratory Syndrome Coronavirus 2 Infection in 10 Communities, Qatar
}

Andrew Jeremijenko, Hiam Chemaitelly, Houssein H. Ayoub, Moza Alishaq, Abdul-Badi Abou-Samra, Jameela Ali A.A. Al Ajmi, Nasser Ali Asad Al Ansari, Zaina Al Kanaani, Abdullatif Al Khal, Einas Al Kuwari,

Ahmed Al-Mohammed, Naema Hassan Abdulla Al Molawi, Huda Mohamad Al Naomi, Adeel A. Butt, Peter Coyle, Reham Awni El Kahlout, Imtiaz Gillani, Anvar Hassan Kaleeckal, Naseer Ahmad Masoodi, Anil George Thomas, Hanaa Nafady-Hego, Ali Nizar Latif, Riyazuddin Mohammad Shaik, Nourah B.M. Younes, Hanan F. Abdul Rahim, Hadi M. Yassine, Mohamed G. Al Kuwari, Hamad Eid Al Romaihi, Mohamed H. Al-Thani, Roberto Bertollini, Laith J. Abu-Raddad

We investigated what proportion of the population acquired severe acute respiratory syndrome coronavirus 2 (SARSCoV-2) infection and whether the herd immunity threshold has been reached in 10 communities in Qatar. The study included 4,970 participants during June 21-September 9 , 2020. Antibodies against SARS-CoV-2 were detected by using an electrochemiluminescence immunoassay. Seropositivity ranged from $54.9 \%(95 \% \mathrm{Cl} 50.2 \%-59.4 \%)$ to $83.8 \%(95 \% \mathrm{Cl} 79.1 \%-87.7 \%)$ across communities and showed a pooled mean of $66.1 \%$ (95\% Cl 61.5\%-70.6\%). A range of other epidemiologic measures indicated that active infection is rare, with limited if any sustainable infection transmission for clusters to occur. Only 5 infections were ever severe and 1 was critical in these young communities; infection severity rate of $0.2 \%(95 \% \mathrm{Cl} 0.1 \%-0.4 \%)$. Specific communities in Qatar have or nearly reached herd immunity for SARS-CoV-2 infection: $65 \%-70 \%$ of the population has been infected.

Since the start of the severe acute respiratory synSdrome coronavirus 2 (SARS-CoV-2) pandemic, millions of infections have been confirmed through real-time reverse transcription PCR (RT-PCR) testing

Author affiliations: Hamad Medical Corporation, Doha, Qatar (A. Jeremijenko, M. Alishaq, A.-B. Abou-Samra, J.A.A.A. Al Ajmi, N.A.A. Al Ansari, Z. Al Kanaani, A. Al Khal, E. Al Kuwari, A. Al-Mohammed, N.H.A. Al Molawi, H.M. Al Naomi, A.A. Butt, P. Coyle, R.A. El Kahlout, I. Gillani, A.H. Kaleeckal, N.A. Masoodi, A.G. Thomas, H. Nafady-Hego, A.N. Latif, R.H. Shaik, N.B.M. Younes); Weill Cornell Medicine-Qatar of Cornell University, Doha (H. Chemaitelly, L.J. Abu-Raddad); World Health Organization Collaborating Centre for Disease Epidemiology
(1), and millions probably have gone undocumented (2). Two key questions remain unanswered. Has any community reached herd immunity to render infection transmission chains unsustainable? What proportion of the population needs to be infected to reach herd immunity?

Qatar, a peninsula in the Arabian Gulf region that has a diverse population of 2.8 million (3), has experienced a large-scale SARS-CoV-2 epidemic $(4,5)$. By January 14, 2021, the rate of real-time RT-PCR-confirmed infections exceeded 65 cases/1,000 persons (6). The epidemic, which is currently in an advanced stage (4), seems to have followed a classic susceptibleinfected-recovered pattern, with an epidemic peak around May 20, followed by a steady decrease for the next 8 months (4).

The subpopulation most affected by this epidemic was expatriate craft and manual workers (CMWs) among whom community transmission was first identified (4). These workers constitute $\approx 60 \%$ of the population in Qatar and are typically single men 20-49 years of age (7). CMWs at a given workplace

Analytics on HIVIAIDS, Sexually Transmitted Infections, Doha (H. Chemaitelly, L.J. Abu-Raddad); Qatar University, Doha (H.H. Ayoub, H.F. Abdul Rahim, H.M. Yassine); Assiut University, Assiut, Egypt (H. Nafady-Hego); Primary Health Care Corporation, Doha (M.G. Al Kuwari); Ministry of Public Health, Doha (H.E. Al Romaihi, M.H. Al-Thani, R. Bertollini); Cornell University, New York, New York, USA (A.A. Butt, L.J. Abu-Raddad)

DOI: https://doi.org/10.3201/eid2705.20.4365 
or company not only work together during the day but also live together as a community in large dormitories or housing complexes in which they share rooms, bathrooms, and cafeteria-style meals $(4,8)$. These communities stay mostly in contact with their own community members and infrequently mingle with other communities, creating a geographic bubble that proved essential for the pattern of infection transmission (4). With reduced options for effective social and physical distancing, SARS-CoV-2 transmission in these CMW communities resembled that of influenza outbreaks in schools $(4,9,10)$, and especially boarding schools (10). This finding is observed despite implementation of nonpharmaceutical control measures, such as a mask mandate after the World Health Organization (WHO) recommendation (11), promotion and facilitation of social and physical distancing, disinfection of surfaces, and awareness messaging in different languages. A similar transmission pattern has been documented among migrant workers in Singapore $(12,13)$ and Spain (14).

Factors observed included the large number of diagnosed infections in CMWs (4), the large proportion of infections that were asymptomatic $(4,15,16)$, the high real-time RT-PCR positivity rates in the random testing campaigns conducted around the epidemic peak in different CMW communities (4), the observed susceptible-infected-recovered epidemic curve with steady decreases in incidence for 8 months despite the gradual easing of the social and physical distancing restrictions $(4,17)$, and evidence indicating an efficacy $>90 \%$ for natural infection against reinfection that lasts for $\geq 7$ months (18; L.J. Abu-Raddad et al., unpub. data). All of these factors raised questions of whether herd immunity might have been reached in at least some of these communities.

On the basis of these considerations, we hypothesized that at least some of the CMW communities have already reached the herd immunity threshold. To investigate this hypothesis, our specific objective was to assess the proportion of the population that has been infected by assessing the level of detectable antibodies. More than $90 \%$ of real-time RT-PCR-confirmed cases in Qatar show development of detectable antibodies (4); therefore, we operationally defined herd immunity as the proportion of the population that needs to have detectable antibodies before infection transmission/circulation becomes unsustainable in this population, with limited if any new infections occurring. The study was conducted to inform the national response and preparedness for potential future infection waves.

\section{Methods}

\section{Data Sources}

We conducted testing for detectable SARS-CoV-2specific antibodies in blood specimens in $10 \mathrm{CMW}$ communities during June 21-September 9, 2020. This testing was part of an a priori-designed study combined with a testing and surveillance program led by the Ministry of Public Health and Hamad Medical Corporation (HMC), the main public healthcare provider in Qatar and the nationally designated provider for all COVID-19 healthcare needs. The goal of this program was to assess the level of infection exposure in different subpopulations and economic sectors.

The study design was opportunistic using the Ministry of Public Health-HMC program and the need for rapid data collection to inform the national response. We specifically selected the $10 \mathrm{CMW}$ communities for feasibility or given earlier random real-time RT-PCR testing campaigns or contact tracing that suggested substantial infection levels. For instance, CMW community 1 was part of a random real-time RT-PCR testing campaign that identified, by using nasopharyngeal swab specimens, a high positivity rate of 59\% during late April 2020.

The population size of each of these communities ranged from a few hundred to a few thousand who live in shared accommodations provided by the employers. The companies that employ these workers belonged to the service or industrial sectors, but the bulk of the employees, even in the industrial companies, worked on providing services, such as catering, cleaning, and other janitorial services, warehousing, security, and port work.

Ten employers were contacted and were willing to participate and advertise the availability and location of testing sites to their employees. Participation was voluntary. Employees interested in being tested and in knowing their status were provided with transportation to HMC testing sites. Informed consent was able to be obtained in 9 languages (Arabic, Bengali, English, Hindi, Urdu, Nepali, Sinhala, Tagalog, and Tamil) to cater to the main language groups spoken in the CMW communities of Qatar.

We used self-administered questionnaires in these same languages only for CMW community 1 ; questionnaires were given by trained public health workers to collect data on sociodemographics and history of exposure and symptoms. We developed the questionnaire on the basis of suggestions from WHO (19). A blood specimen was obtained from all study participants, and in 6 communities, nasopharyngeal swab specimens were simultaneously collected for 
real-time RT-PCR testing by licensed nurses. We applied national guidelines and standard of care to all identified real-time RT-PCR-positive case-patients, including requirement of isolation and other measures to prevent infection transmission. No action was mandated by the national guidelines to those persons found to be antibody positive but real-time RT-PCR negative, and thus no action was taken apart from notifying persons of their serostatus.

We subsequently linked results of the serologic testing to the HMC centralized and standardized database comprising all SARS-CoV-2 real-time RT-PCR testing conducted in Qatar since the start of the epidemic (4). The database also includes data on hospitalization and on the WHO severity classification (20) for each real-time RT-PCR-confirmed infection. Data were also linked to datasets of 2 recently completed national reinfection studies (18; L.J. Abu-Raddad et al., unpub. data) to identify reinfections. The study was approved by HMC and Weill Cornell MedicineQatar Institutional Review Boards.

\section{Laboratory Methods}

We performed testing for SARS-CoV-2-specific antibodies in serologic samples by using an electrochemiluminescence immunoassay (Roche Elecsys AntiSARS-CoV-2, https://www.roche.com) (sensitivity $99.5 \%$, specificity $99.8 \%)(21,22)$. We interpreted results according to the manufacturer's instructions: reactive for a cutoff index $\geq 1.0$ and nonreactive for a cutoff index $<1.0$ (22).

We performed real-time RT-PCR testing of aliquots of universal transport medium (Huachenyang Technology, https://szhcy.en.alibaba.com) used for collection of nasopharyngeal swab specimens. We extracted aliquots by using the QIAsymphony Platform (QIAGEN, https://www.qiagen.com); tested them by using real-time PCR (TaqPath COVID-19 Combo Kit; Thermo Fisher Scientific, https://www.thermofisher.com (sensitivity 100\%, specificity 100\%) (23) in an ABI 7500 FAST System (ThermoFisher); extracted them by using a custom protocol (M.K. Kalikiri et al., Sidra Medicine, pers. comm., 2021 Feb 1) on a Hamilton Microlab STAR (https:/ / www.hamiltoncompany.com); tested them by using the AccuPower SARSCoV-2 Real-Time RT-PCR Kit (Bioneer, https:/ / www. bioneer.com) (sensitivity $100 \%$, specificity 100\%) (24) on an ABI 7500 FAST System or loaded them directly into a Roche Cobas 6800 system; and assayed them by using the Cobas SARS-CoV-2 Test (sensitivity $95 \%$, specificity 100\%) (25). All laboratory testing was conducted at HMC Central Laboratory following standardized protocols.

\section{Statistical Analysis}

We used frequency distributions to describe characteristics of CMWs and to estimate different SARSCoV-2 epidemiologic measures. We estimated the pooled mean for SARS-CoV-2 seropositivity across CMW communities by using meta-analysis. We applied a DerSimonian-Laird random-effects model (26) to pool seroprevalence measures that were weighted by using the inverse-variance method $(27,28)$.

We used $\chi^{2}$ tests and univariable logistic regressions to determine the association of each prespecified covariate (i.e., sex, age, nationality, and CMW community) with seropositivity. For CMW community 1, we also investigated associations of educational attainment, contact with an infected person, presence of symptoms in the previous 2 weeks, and whether symptoms required medical attention with seropositivity. Missing values were included as separate subcategories in the analyses. We generated summary statistics, as well as odds ratios (ORs), 95\% CIs, and p values (Tables 1, 2; Appendix Tables 1, 2, https://wwwnc.cdc.gov/EID/ article/27/5/20-4365-App1.pdf).

We performed multivariable logistic regressions to estimate the magnitude of the association of a specific covariate adjusting for other covariates in the model. Covariates with $\mathrm{p}$ values $\leq 0.2$ in univariable regression analysis were included simultaneously in the multivariable logistic regression model. Covariates with $p$ values $\leq 0.05$ in the multivariable model were considered as showing evidence for an association with the outcome, and associated adjusted ORs (aORs), 95\% CIs, and $\mathrm{p}$ values were generated and reported (Tables 1, 2; Appendix Tables 1, 2). No interactions were investigated. Statistical models' goodness of fit were reported. The distribution of real-time RTPCR cycle threshold $\left(C_{t}\right)$ values for persons who were real-time RT-PCR positive was further generated, and summary statistics were reported. Statistical analyses were performed by using STATA/SE version 16.1 (https://www.stata.com) (29).

We also conducted mathematical modeling simulations to highlight the effect of heterogeneity in the risk for exposure to the infection on the level of herd immunity. These simulations were generated by using a classic age-structured, susceptibleexposed-infectious-recovered mathematical model published elsewhere (17). Simulations were implemented by using MATLAB R2019a (https://www. mathworks.com) (30).

\section{Results}

A total of 4,970 CMWs from the $10 \mathrm{CMW}$ communities participated in this study (Table 1). Participants 
Table 1. Characteristics of $10 \mathrm{CMWs}$ and associations with SARS-CoV-2 seropositivity, indicated by detectable antibodies in serologic samples, Qatar*

\begin{tabular}{|c|c|c|c|c|c|c|c|c|}
\hline \multirow[b]{2}{*}{ Characteristic } & \multirow{2}{*}{$\begin{array}{l}\text { No. }(\%) \dagger \\
\text { tested }\end{array}$} & \multicolumn{3}{|c|}{ SARS-CoV-2 seropositive } & \multicolumn{2}{|c|}{$\begin{array}{c}\text { Univariable regression } \\
\text { analysis }\end{array}$} & \multicolumn{2}{|c|}{$\begin{array}{c}\text { Multivariable regression } \\
\text { analysis } \ddagger\end{array}$} \\
\hline & & No. & $\% \S(95 \% \mathrm{Cl})$ & $\mathrm{p}$ value & OR $(95 \% \mathrm{Cl})$ & $p$ valueף & OR $(95 \% \mathrm{Cl})$ & $\mathrm{p}$ value\# \\
\hline \multicolumn{9}{|l|}{ Sex } \\
\hline $\mathrm{M}$ & ,721 (95.0) & 153 & $66.8(65.4-68.1)$ & $<0.001$ & Referent & & Referent & \\
\hline $\mathrm{F}$ & & 46 & $105(1202201$ & & $11(0.08-0$. & $<0.001$ & $0.13(0.09-0$ & $<0.001$ \\
\hline \multicolumn{9}{|l|}{ Age, $y$} \\
\hline$<29$ & $1,579(31.8)$ & 1,031 & $65.3(62.9-67.6)$ & $<0.001$ & Referent & & Referent & \\
\hline $30-39$ & 1,973 (39.7) & 1,226 & $62.1(60.0-64.3)$ & & $0.87(0.76-1.00)$ & 0.052 & $0.90(0.78-1.05)$ & 0.178 \\
\hline $40-49$ & $1,040(20.9)$ & 680 & $65.4(62.4-68.3)$ & & $1.00(0.85-1.18)$ & 0.962 & $1.12(0.93-1.35)$ & 0.216 \\
\hline$\geq 50$ & $339(6.8)$ & 225 & $66.4(61.1-71.4)$ & & $1.05(0.82-1.34)$ & 0.705 & $1.21(0.92-1.59)$ & 0.170 \\
\hline Missing & $39(0.8)$ & 37 & $94.9(82.7-99.4)$ & & $9.83(2.36-40.95)$ & 0.002 & $9.57(2.22-41.32)$ & 0.002 \\
\hline \multicolumn{9}{|l|}{ Nationality } \\
\hline Other** & $125(2.5)$ & 40 & $32.0(23.9-40.9)$ & $<0.001$ & Referent & & Referent & \\
\hline Filipino & $186(3.7)$ & 68 & $36.6(29.6-43.9)$ & & $1.22(0.76-1.98)$ & 0.408 & $2.23(1.32-3.75)$ & 0.003 \\
\hline Sri Lankan & $147(3.0)$ & 77 & $52.4(44.0-60.7)$ & & $2.34(1.42-3.84)$ & 0.001 & $2.81(1.66-4.76)$ & $<0.001$ \\
\hline Kenyan & $152(3.1)$ & 77 & 50.7 (42.4-58.9) & & 2.18 (1.33-3.57) & 0.002 & $3.43(1.99-5.90)$ & $<0.001$ \\
\hline Indian & $1,647(33.1)$ & 1,035 & $62.8(60.5-65.2)$ & & $3.59(2.44-5.30)$ & $<0.001$ & $3.60(2.40-5.41)$ & $<0.001$ \\
\hline Nepalese & $2,136(43.0)$ & 1,468 & 68.7 (66.7-70.7) & & 4.67 (3.17-6.88) & $<0.001$ & $4.93(3.27-7.42)$ & $<0.001$ \\
\hline Bangladeshi & $577(11.6)$ & 434 & 75.2 (71.5-78.7) & & 6.45 (4.23-9.82) & $<0.001$ & $6.78(4.31-10.66)$ & $<0.001$ \\
\hline \multicolumn{9}{|l|}{ CMW community } \\
\hline 5 & 443 (8.9) & 243 & $54.9(50.1-59.6)$ & $<0.001$ & Referent & & Referent & \\
\hline 4 & $534(10.7)$ & 330 & $61.8(57.5-65.9)$ & & 1.33 (1.03-1.72) & 0.028 & $1.12(0.83-1.52)$ & 0.449 \\
\hline 10 & 957 (19.3) & 620 & $64.8(61.7-67.8)$ & & $1.51(1.20-1.90)$ & $<0.001$ & $1.30(1.02-1.65)$ & 0.034 \\
\hline 7 & $188(3.8)$ & 122 & $64.9(57.6-71.7)$ & & $1.52(1.07-2.17)$ & 0.020 & $1.31(0.91-1.89)$ & 0.154 \\
\hline 6 & $1,505(30.3)$ & 946 & $62.9(60.4-65.3)$ & & 1.39 (1.12-1.73) & 0.002 & $1.32(1.06-1.66)$ & 0.015 \\
\hline 2 & $456(9.2)$ & 282 & $61.8(57.2-66.3)$ & & $1.33(1.02-1.74)$ & 0.034 & $1.46(1.08-1.96)$ & 0.013 \\
\hline 9 & $202(4.1)$ & 126 & $62.4(55.3-69.1)$ & & $1.36(0.97-1.92)$ & 0.074 & $1.71(1.18-2.48)$ & 0.005 \\
\hline 8 & 139 (2.8) & 93 & $66.9(58.4-74.6)$ & & $1.66(1.12-2.48)$ & 0.013 & $1.92(1.25-2.95)$ & 0.003 \\
\hline 1 & $255(5.1)$ & 193 & $75.7(69.9-80.8)$ & & $2.56(1.82-3.61)$ & $<0.001$ & $2.52(1.75-3.62)$ & $<0.001$ \\
\hline 3 & $291(5.9)$ & 244 & $83.8(79.1-87.9)$ & & $4.27(2.97-6.15)$ & $<0.001$ & $3.49(2.41-5.07)$ & $<0.001$ \\
\hline \multicolumn{9}{|c|}{$\begin{array}{l}{ }^{*} \text { CMW, craft and manual worker; OR, odds ratio; SARS-CoV-2, severe acute respiratory syndrome coronavirus } 2 . \\
\text { †Percentage of total sample. } \\
\text { fPseudo-R }{ }^{2} \text { value in the multivariable logistic regression model is } 7.1 \% \text {. } \\
\text { \$Percent seropositive of those tested. } \\
\text { TCovariates with } \mathrm{p} \leq 0.2 \text { in univariable analysis (i.e., sex, age, nationality, and CMW community) were included in the multivariable analysis. } \\
\text { \#Covariates with } \mathrm{p} \leq 0.05 \text { in multivariable analysis (i.e., sex, nationality, and CMW community) were considered predictors of SARS-CoV-2 seropositivity. } \\
\text { **Includes all other nationalities that contributed < } 10 \% \text { of the sample in each community. These are: Canadian, Egyptian, Ethiopian, Georgian, Ghanaian, } \\
\text { Indonesian, Iraqi, Jordanian, Lebanese, Nigerian, Pakistani, Palestinian, Somali, Tanzanian, Tunisian, Ugandan, and Yemeni. }\end{array}$} \\
\hline
\end{tabular}

were mostly men $(95.0 \%) ;<40$ years of age $(71.5 \%)$; and of Nepalese $(43.0 \%)$, Indian $(33.1 \%)$, or Bangladeshi $(11.6 \%)$ origin. Regression analyses identified each of sex, nationality, and CMW community to be independently associated with seropositivity.

Women had $87 \%$ lower odds of being seropositive than men (aOR 0.13, 95\% CI 0.09-0.19) (Table 1). Compared with all other nationalities (Table 1), aOR was 6.78 (95\% CI 4.31-10.66) for Bangladeshis, 4.93 (95\% CI 3.27-7.42) for Nepalese, 3.60 (95\% CI 2.405.41) for Indians, 3.43 (95\% CI 1.99-5.90) for Kenyans, 2.81 (95\% CI 1.66-4.76) for Sri Lankans, and 2.23 (95\% CI 1.32-3.75) for Filipinos. Some differences in seropositivity by CMW community were noted (Table 1). No major differences in seropositivity by age group were found (Table 1).

We provide characteristics and associations with seropositivity (detectable antibodies in serologic samples) for only CMW community 1 , in which a specific self-administered questionnaire was administered and collected specific sociodemographic data and history of exposure and symptoms (Table 2). Nearly $40 \%$ of participants had intermediate or low educational attainment, and $31 \%$ had higher schooling levels or vocational training. University education was associated with a $75 \%$ (OR 0.25, 95\% CI 0.09-0.67) lower odds of seropositivity compared with intermediate or lower educational attainment. No significant associations with seropositivity were found for contact with an infected person, presence of symptoms, or symptoms requiring medical attention. We provide characteristics and associations with SARS-CoV-2 seropositivity for CMW communities 2-10 (Appendix Table 1). For each of these communities, associations were found for sex and nationality, but no major associations were found for age group.

We provide key SARS-CoV-2 epidemiologic measures in the different CMW communities (Figure 1). Of 4,970 SARS-CoV-2 antibody test results for these CMWs, 3,199 $(64.4 \%$, 95\% CI $63.0 \%-65.7 \%)$ were 
seropositive. Seropositivity ranged from 54.9\% (95\% CI $50.2 \%-59.4 \%$ ) for CMW community 5 to $83.8 \%$ (95\% CI 79.1\%-87.7\%) for CMW community 3 (Figure 1 , panel A). The pooled mean for SARS-CoV-2 seropositivity across the $10 \mathrm{CMW}$ communities was $66.1 \%$ (95\% CI 61.5\%-70.6\%).

Of 2,016 real-time RT-PCR tests using nasopharyngeal swab specimens collected during this study for these CMWs, 112 (5.6\%, 95\% CI 4.6\%-6.6\%) were positive. Real-time RT-PCR positivity ranged from $0.0 \%$ (95\% CI $0.0 \%-3.9 \%$ ) for CMW community 1 and $0.0 \%(95 \%$ CI $0.0 \%-9.0 \%)$ for CMW community 8 to 10.5\% (95\% CI 7.4\%-14.8\%) for CMW community 3 (Figure 1, panel B). Pooled mean real-time RT-PCR positivity across the $6 \mathrm{CMW}$ communities in which real-time RT-PCR testing was conducted was 3.9\% (95\% CI $1.6 \%-6.9 \%)$. The $\mathrm{C}_{\mathrm{t}}$ values ranged from 15.8 to
37.4 (median 34.0) (Figure 2). Most (79.5\%) real-time RT-PCR-positive persons had $C_{t}$ values $>30$, suggestive of no active infection $(31,32)$. Major differences in real-time RT-PCR positivity were found by nationality and CMW community (Appendix Table 2).

Infection positivity (antibody or real-time RT-PCR positive) ranged from $62.5 \%$ (95\% CI $58.3 \%-66.7 \%)$ for CMW community 4 to $83.8 \%$ (95\% CI $79.1 \%-87.7 \%$ ) for CMW community 3 (Figure 1, panel C). Pooled mean infection positivity across the $6 \mathrm{CMW}$ communities with antibody and RT-PCR results was $69.5 \%$ (95\% CI 62.8\%-75.9\%).

Data were linked to the national SARS-CoV-2 realtime RT-PCR testing and hospitalization database. Of the 3,199 antibody-positive CMWs, 1,012 (31.6\%, 95\% CI $30.0 \%-33.3 \%$ ) were previously given a diagnosis of SARS-CoV-2 infection (had a record of a real-time

Table 2. Characteristics of CMW community 1 and associations with SARS-CoV-2 seropositivity (detectable antibodies in serologic samples) including sociodemographics, history of exposure, and symptoms, Qatar*

\begin{tabular}{|c|c|c|c|c|c|c|}
\hline \multirow[b]{2}{*}{ Characteristic } & \multirow[b]{2}{*}{ No. $(\%) \dagger$ tested } & \multicolumn{3}{|c|}{ SARS-CoV-2 seropositive } & \multicolumn{2}{|c|}{$\begin{array}{c}\text { Univariable regression } \\
\text { analysis } \ddagger\end{array}$} \\
\hline & & No. & $\% \S(95 \% \mathrm{Cl})$ & $p$ value & OR $(95 \% \mathrm{Cl})$ & $p$ value \\
\hline \multicolumn{7}{|l|}{ Sex } \\
\hline M & $240(94.1)$ & 189 & 78.8 (73.0-83.7) & \multirow[t]{2}{*}{$<0.001$} & Referent & \\
\hline $\mathrm{F}$ & $15(5.9)$ & 4 & $26.7(7.8-55.1)$ & & $0.10(0.03-0.32)$ & $<0.001$ \\
\hline \multicolumn{7}{|l|}{ Age, y } \\
\hline$<29$ & $105(41.2)$ & 84 & $80.0(71.1-87.2)$ & \multirow[t]{4}{*}{0.322} & Referent & \\
\hline 30-39 & $111(43.5)$ & 83 & $74.8(65.6-82.5)$ & & $0.74(0.39-1.41)$ & 0.360 \\
\hline $40-49$ & $27(10.6)$ & 19 & $70.4(49.8-86.2)$ & & $0.59(0.23-1.54)$ & 0.284 \\
\hline$\geq 50$ & $12(4.7)$ & 7 & $58.3(27.7-84.8)$ & & $0.35(0.10-1.21)$ & 0.098 \\
\hline \multicolumn{7}{|l|}{ Nationality } \\
\hline Other\# & $48(18.8)$ & 23 & $47.9(33.3-62.8)$ & \multirow[t]{4}{*}{$<0.001$} & Referent & \\
\hline Indian & $32(12.5)$ & 20 & 62.5 (43.7-78.9) & & $1.81(0.73-4.51)$ & 0.202 \\
\hline Nepalese & $157(61.6)$ & 132 & 84.1 (77.4-89.4) & & $5.74(2.82-11.67)$ & $<0.001$ \\
\hline Bangladeshi & $18(7.1)$ & 18 & $100.0(81.5-100.0)$ & & Omitted by model & NA \\
\hline \multicolumn{7}{|l|}{ Education level } \\
\hline Intermediate or lower & $101(39.6)$ & 88 & $87.1(79.0-93.0)$ & \multirow[t]{4}{*}{$<0.001$} & Referent & \\
\hline Secondary/high school/vocational & $80(31.4)$ & 69 & $86.3(76.7-92.9)$ & & $0.93(0.39-2.20)$ & 0.863 \\
\hline University & $27(10.6)$ & 17 & $63.0(42.4-80.6)$ & & $0.25(0.09-0.67)$ & 0.005 \\
\hline Missing & $47(18.4)$ & 19 & $40.4(26.4-55.7)$ & & $0.10(0.04-0.23)$ & $<0.001$ \\
\hline \multicolumn{7}{|l|}{ Contact with an infected person } \\
\hline No & $124(48.6)$ & 93 & $75.0(66.4-82.3)$ & \multirow[t]{3}{*}{0.303} & Referent & \\
\hline Yes & $14(5.5)$ & 13 & $92.9(66.1-99.8)$ & & $4.33(0.54-34.48)$ & 0.166 \\
\hline Unknown/missing & 117( & 87 & $74.4(65.5-82.0)$ & & $0.97(0.54-1.73)$ & 0.909 \\
\hline \multicolumn{7}{|l|}{ Symptoms in the past 2 weeks $^{* *}$} \\
\hline Asymptomatic & $184(72.2)$ & 148 & $80.4(74.0-85.9)$ & \multirow[t]{4}{*}{$<0.001$} & Referent & \\
\hline 1 & $16(6.3)$ & 16 & $100.0(79.4-100.0)$ & & Omitted by model & \\
\hline$>2$ & $12(4.7)$ & 12 & $100.0(73.5-100.0)$ & & Omitted by model & NA \\
\hline Missing & $43(16.9)$ & 17 & $39.5(25.0-55.6)$ & & $0.16(0.08-0.32)$ & $<0.001$ \\
\hline \multicolumn{7}{|l|}{ Symptoms required medical attention } \\
\hline No & $210(82.4)$ & 174 & $82.9(77.1-87.7)$ & \multirow[t]{3}{*}{$<0.001$} & Referent & \\
\hline Yes & $3(1.2)$ & 3 & $100.0(29.2-100.0)$ & & mitted by model & NA \\
\hline Unknown/missing & $42(16.5)$ & 16 & $38.1(23.6-54.4)$ & & $0.13(0.06-0.26)$ & $<0.001$ \\
\hline \multicolumn{7}{|c|}{$\begin{array}{l}\text { *CMW, craft and manual worker; NA, not applicable; OR, odds ratio; SARS-CoV-2, severe acute respiratory syndrome coronavirus } 2 . \\
\text { †Percentage of total sample. } \\
\text { fOverall sample size and numbers per stratum were too small to warrant conduct of meaningful multivariable regression analysis. } \\
\text { §Percent seropositive of those tested. } \\
\text { TCovariates with } p \leq 0.05 \text { in univariable analysis (i.e., sex, nationality, and education level were considered as showing evidence for an association with } \\
\text { SARS-CoV-2 seropositivity. }\end{array}$} \\
\hline
\end{tabular}


RT-PCR-confirmed positive result before this study). No records of previous real-time RT-PCR positive test results were found for the remaining 2,187 antibodypositive CMWs. For the CMW communities that were previously part of broad real-time RT-PCR testing because of a case identification or a random testing campaign, the diagnosis rate ranged from $28.0 \%$ (95\% CI $19.1 \%-38.2 \%$ ) for CMW community 8 to $82.9 \%$ (95\% CI $76.8 \%-87.9 \%$ ) for CMW community 1 . In instances in which no such broad real-time RT-PCR testing was conducted, the diagnosis rate was only $13.2 \%$ (95\% CI $10.7 \%-16.1 \%)$ for CMW community $10,7.4 \%(95 \%$ CI $4.7 \%-11.2 \%$ ) for CMW community 2 , and $0.4 \%$ (95\% CI $0.0 \%-2.3 \%)$ for CMW community 3. Only a small fraction of antibody-negative persons, 14 of $1,771(0.8 \%, 95 \%$ CI $0.4 \%-1.3 \%)$, had been previously given a diagnosis of being real-time RT-PCR positive (Appendix Table 3).
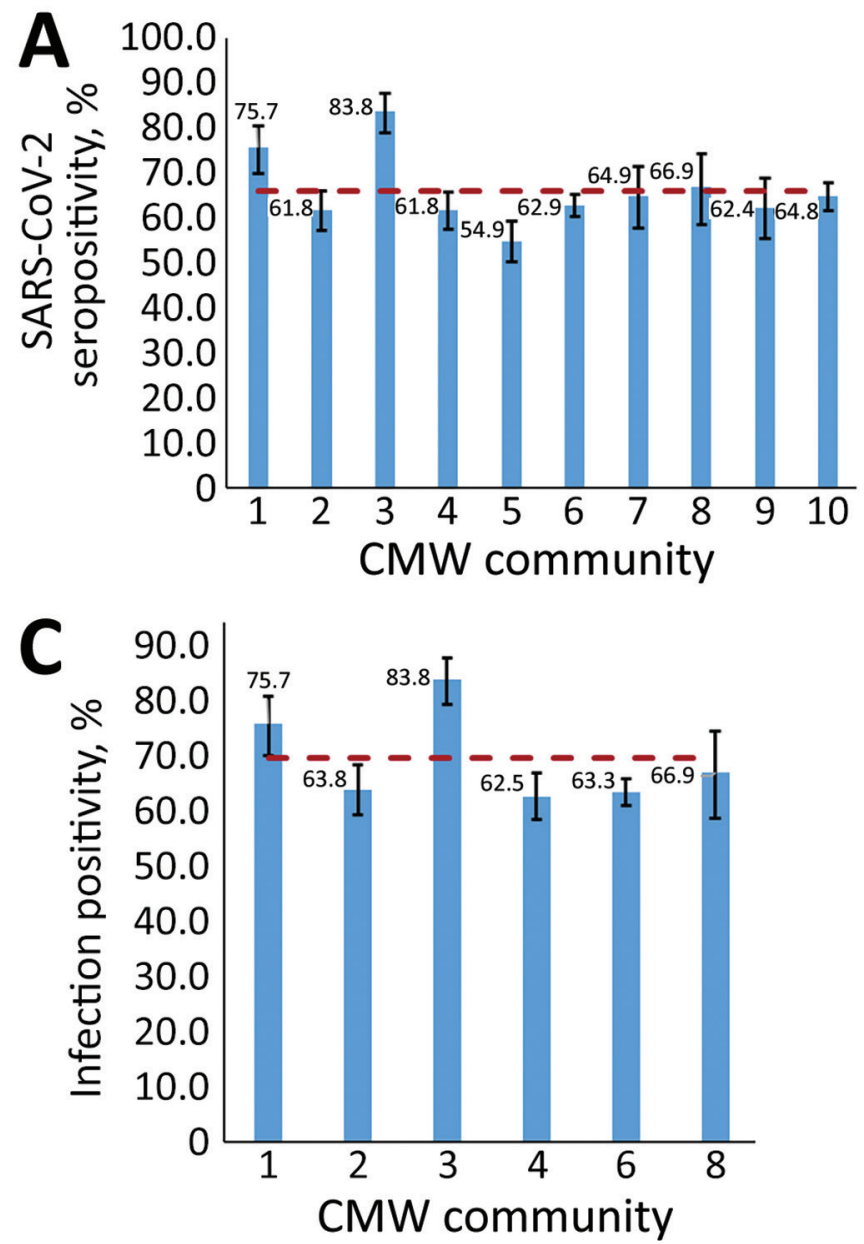

Of the total sample, 21 persons had a hospitalization record associated with a SARS-CoV-2 infection diagnosis, of whom, infection severity per WHO classification was mild for 5 , moderate for 10 , severe for 5 , and critical for 1 . All 21 persons eventually cleared their infection and were discharged from the hospital. All of these persons were also antibody positive. Accordingly, the proportion of those persons who had a confirmed severe or critical infection of the 3,233 persons who had a laboratory-confirmed infection (antibody or real-time RT-PCR positive result) was $0.2 \%$ (95\% CI 0.1\%-0.4\%).

We linked our data to records of 2 recently completed studies. These studies, which assessed reinfection in Qatar in a cohort of $>130,000$ real-time RTPCR-confirmed infected persons (18) and a cohort of $>43,000$ antibody-positive persons (L.J. Abu-Raddad et al., unpub. data), identified no reinfections in these
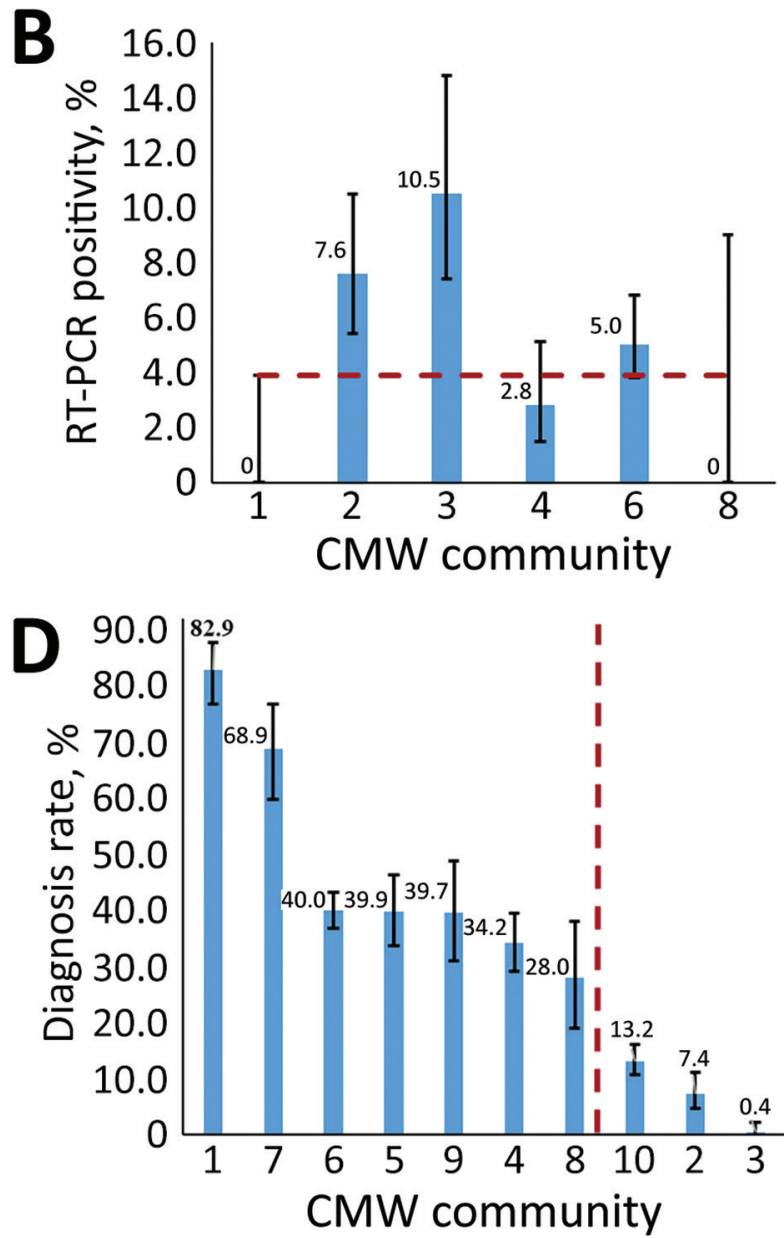

Figure 1. Measures of SARS-CoV-2 infection across 10 craft and manual worker communities, Qatar. A) Seropositivity (antibody positivity), B) real-time RT-PCR positivity, C) infection positivity (antibody or real-time RT-PCR positive), and D) diagnosis rate. Panels B and $\mathrm{D}$ show results for only the 6 communities for whom real-time RT-PCR testing was performed. Percentages are shown above bars. Numbers along the x-axes of each panel indicate the community number. Error bars indicate $95 \% \mathrm{Cls}$. CMW, craft and manual workers; RT-PCR, real-time reverse transcription PCR; SARS-CoV-2, severe acute respiratory syndrome coronavirus 2. 
study participants whose results were confirmed by using viral genome sequencing.

\section{Discussion}

Our results support that herd immunity has been reached (or at least nearly reached) in these CMW communities, and that the level of herd immunity needed for SARS-CoV-2 infection is a proportion of the population infected of $\approx 65 \%-70 \%$. This conclusion has been reached considering the following lines of evidence. First, these CMW communities had comparable seroprevalences of $\approx 65 \%-70 \%$. Second, realtime RT-PCR positivity was low and most of those who were real-time RT-PCR positive had a high $C_{t}$ suggestive of an earlier rather than recent infection $(31,32)$. Third, only a few persons had active infection $\left(C_{t}<25\right)$ and no major infection cluster was identified in any of these CMW communities during this study or thereafter (suggestive of isolated infections and unsustainable infection transmission for clusters to occur). Fourth, 2 recent studies from Qatar reported an efficacy $>90 \%$ for natural infection against reinfection for $\geq 7$ months after primary infection (18; L.J. Abu-Raddad et al., unpub. data), in addition to other evidence on the durability of immunity (33-35). Fifth, the level of $65 \%-70 \%$ infection exposure is in concordance with that predicted by using the classical formula for herd immunity of $1-1 / R_{0}(36,37) ; R_{0}$, the basic reproduction number, as $2.5-4.0(38,39)$.

Although large clusters of infection were common in such CMW communities before and around the epidemic peak toward end of May, that time is several weeks before the launch of this study. Thus, no such major cluster has been subsequently identified in such CMW communities in Qatar, despite the progressive easing of the social and physical distancing restrictions since June 15, 2020.

These findings indicate that reaching herd immunity in such largely homogenous communities requires high exposure levels of $\approx 65 \%-70 \%$. However, true herd immunity might have been reached even at a lower proportion of the population infected. Mathematical modeling indicates that infection exposure for a novel infection (especially in the first cycle) can considerably overshoot the classical herd immunity level of $1-1 / R_{0^{\prime}}$ more so if the social contact rate within this community is homogeneous (Appendix Figure 1). Heterogeneity in the social contact rate can reduce the final proportion of the population that needs to be infected to reach herd immunity (Appendix Figure 1) (37; R. Aguas et al., Oxford University, pers. comm., 2021 Feb 1).

The severity rate for SARS-CoV-2 infection was low $(0.2 \%)$, possibly because of the young age of the

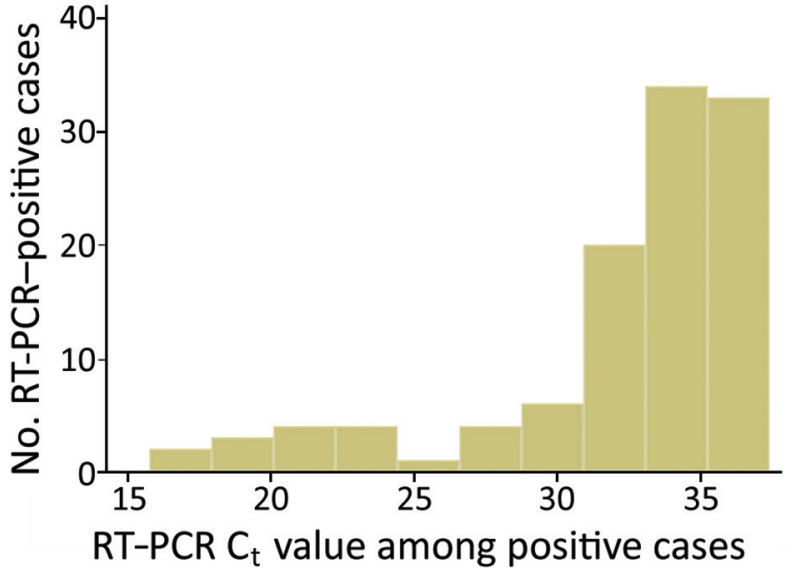

Figure 2. Distribution of real-time RT-PCR $C_{t}$ values among craft and manual workers identified as real-time RT-PCR positive for severe acute respiratory syndrome coronavirus 2, Qatar. $C_{t}$, cycle threshold; RT-PCR, real-time reverse transcription PCR.

CMWs. No COVID-19 deaths were reported in these CMW communities. In communities in which no previous, broad real-time RT-PCR testing was conducted, $<15 \%$ of the antibody-positive persons had ever been given a diagnosis as being real-time RT-PCR positive before this study. There was a large difference in infection exposure between women and men (Table 1). This difference, with the variable proportion of women across these communities, also explains part of the variation seen in the overall seroprevalence across these communities (Figure 1; Appendix Figure 2). This finding might be attributed to women and men living in different housing accommodations and having different work roles. Women, a small minority in these CMW communities, live in small shared accommodations as opposed to the large ones hosting men.

Differences in results by nationality (Table 1), are explained by nearly all Bangladeshis and Nepalese and most Indians being the workers in these communities, because a proportion of Indians and much of the other nationalities held administrative or managerial positions that had lower social contact rates and possibly lived in different accommodations than most of the workers. No major differences in infection exposure by age were found, although there was a tendency for persons $>40$ years of age to have lower infection exposure (Appendix Table 1), possibly caused by different occupations within these communities.

Our study's limitations included that, by design, the study was specifically conducted in select CMW communities, and therefore findings might not be representative nor generalizable to the wider CMW population in Qatar. The small and variable proportion of women in these communities suggests that 
findings might not also be generalizable to women in these communities. Response rate could not be precisely ascertained given uncertainty around the number of CMWs who were aware of the invitation to participate, but on the basis of employer-reported counts of the size of each community, the response rate was $>50 \%$, and participants expressed high interest in knowing their antibody status. The validity of study outcomes is contingent on the sensitivity and specificity of the used assays. However, laboratory methods were based on high-quality commercial platforms, and each diagnostic method was validated in the laboratory before its use. The antibody assay is one of the best available and extensively used and investigated commercial platforms; it has a specificity $\geq 99.8 \%(22,40,41)$, indicating that falsepositive results, or positive results due to cross-reactivity with other common cold coronaviruses, are not likely.

In conclusion, some of the CMW communities in Qatar, who constituted $\approx 60 \%$ of the total population (7), have reached or nearly reached herd immunity for SARS-CoV-2 infection. Although achieving herd immunity at a national level is difficult within a few months (42), herd immunity could be achieved in specific communities within a few months. In such relatively homogenous communities, reaching herd immunity required infection of $65 \%-70 \%$ of the members of the community. These findings suggest that the SARS-CoV-2 epidemic in a homogenous population is likely to be sustainable until at least two thirds of the population become infected. This finding also suggests that a SARS-CoV-2 vaccine needs at least $65 \%-70 \%$ efficacy at universal coverage for herd immunity to be achieved in a population not exposed to SARS-CoV-2 infection $(43,44 ;$ H.H. Ayoub et al., unpub. data). Alternatively, herd immunity might be reached at a vaccination coverage of $\approx 75 \%$ if vaccine efficacy is $95 \%$, similar to that of the recently licensed SARS-CoV-2 vaccines $(45,46)$.

\section{Acknowledgments}

We thank Hanan Al Kuwari for providing vision, guidance, leadership, and support; Saad Al Kaabi for providing leadership, analytical insights, and an instrumental role in enacting data information systems that made these studies possible; the SWICC Committee and the Scientific Reference and Research Taskforce (SRRT) members for providing informative input, scientific technical advice, and enriching discussions; Mariam Abdulmalik and members of the Tactical Community Command Group on COVID-19 for providing support to the teams that worked on field surveillance;
Nahla Afifi, Tasneem Al-Hamad, Eiman Al-Khayat, and the rest of the Qatar Biobank for Medical Research team for providing unwavering support in retrieving and analyzing samples and in compiling and generating databases for COVID-19 infection; Asmaa Al-Thani for providing leadership; the Clinical Coding Team and the COVID-19 Mortality Review Team (both at Hamad Medical Corporation) and Surveillance Team at the Ministry of Public Health for providing dedicated efforts; Ziad Yehya at Qatargas for providing dedicated logistical and resource support; and all companies that facilitated the participation of their employees in this study.

This study was supported by the Hamad Medical Corporation, Ministry of Public Health, and the Biomedical Research Program and the Biostatistics, Epidemiology, and Biomathematics Research Core, both at Weill Cornell Medicine-Qatar.

A.J. and L.J.A. conceived and designed the study; A.J. collected data; H.C. performed data analyses and wrote the first draft of the manuscript; and H.H.A. contributed to analysis of data. All authors contributed to data acquisition, database development, testing, program development, discussion and interpretation of the results, and writing the manuscript. All authors have read and approved the final manuscript. All data are available in an aggregate form in the main text and Appendix.

\section{About the Author}

Dr. Jeremijenko is a senior consultant in occupational and environmental medicine at Hamad Medical Corporation, Doha, Qatar. His research interests are multidisciplinary, with an emphasis on occupational medicine, toxicology, and infectious diseases.

\section{References}

1. Worldometer. COVID-19 outbreak live update [cited 2020 Sep 6]. https:/ / www.worldometers.info/coronavirus

2. Ioannidis J. Infection fatality rate of COVID-19 inferred from seroprevalence data. Bull World Health Organ. 2020 [cited 2021 Jan 21]. https:/ / www.who.int/bulletin/online_first/ BLT.20.265892.pdf

3. Planning and Statistics Authority. State of Qatar. Qatar monthly statistics [cited 2020 May 26]. https://www.psa.gov.qa/en/pages/default.aspx

4. Abu-Raddad LJ, Chemaitelly H, Ayoub HH, Al Kanaani Z, $\mathrm{Al}$ Khal A, Al Kuwari E, et al. Characterizing the Qatar advanced-phase SARS-CoV-2 epidemic. Sci Rep. 2021;11:623348. https:// doi.org/10.1038/s41598-021-85428-7 .

5. Al Kuwari HM, Abdul Rahim HF, Abu-Raddad LJ, AbouSamra A-B, Al Kanaani Z, Al Khal A, et al.

Epidemiological investigation of the first 5685 cases of SARS-CoV-2 infection in Qatar, 28 February-18 April 2020. BMJ Open. 2020;10:e040428. https:/ / doi.org/10.1136/ bmjopen-2020-040428 
6. Ministry of Public Health. State of Qatar. Coronavirus disease 2019 (COVID-19) [cited 2020 May 25]. https://covid19.moph.gov.qa/EN/Pages/default.aspx

7. Planning and Statistics Authority. State of Qatar. Labor force sample survey [cited 2020 May 1]. https:/ / www.psa. gov.qa/en/statistics/Statistical\%20Releases/Social/ LaborForce/2017/statistical_analysis_labor_force_2017_ En.pdf

8. De Bel-Air F. Demography, migration, and labour market in Qatar [cited 2020 May 1]. https:/ / www.researchgate.net/ publication/323129801_Demography_Migration_and_ Labour_Market_in_Qatar-_UPDATED_June_2017

9. Jackson C, Vynnycky E, Hawker J, Olowokure B, Mangtani P. School closures and influenza: systematic review of epidemiological studies. BMJ Open. 2013;3:e002149. https:/ / doi.org/10.1136/bmjopen-2012-002149

10. Glatman-Freedman A, Portelli I, Jacobs SK, Mathew JI, Slutzman JE, Goldfrank LR, et al. Attack rates assessment of the 2009 pandemic H1N1 influenza A in children and their contacts: a systematic review and meta-analysis. PLoS One. 2012;7:e50228. https:/ / doi.org/10.1371/ journal.pone.0050228

11. Cheng KK, Lam TH, Leung CC. Wearing face masks in the community during the COVID-19 pandemic: altruism and solidarity. Lancet. 2020;Apr 16:[Epub aheqad of print]. https://doi.org/10.1016/S0140-6736(20)30918-1

12. Koh D. Migrant workers and COVID-19. Occup Environ Med.2020;77:634-6. https://doi.org/10.1136/oemed2020-106626

13. Bagdasarian N, Fisher D. Heterogenous COVID-19 transmission dynamics within Singapore: a clearer picture of future national responses. BMC Med. 2020;18:164. https://doi.org/10.1186/s12916-020-01625-7

14. Guijarro C, Pérez-Fernández E, González-Piñeiro B, Meléndez V, José Goyanes M, Renilla ME, et al. Differential risk for COVID-19 in the first wave of the disease among migrants from several areas of the world living in Spain [in Spanish]. Rev Clin Esp. 2020;Nov 20 [Epub ahead of print]. https:// doi.org/10.1016/j.rce.2020.10.10.006.

15. Nikolai LA, Meyer CG, Kremsner PG, Velavan TP. Asymptomatic SARS coronavirus 2 infection: invisible yet invincible. Int J Infect Dis. 2020;100:112-6. https:/ / doi.org/ 10.1016/j.ijid.2020.08.076

16. Oran DP, Topol EJ. Prevalence of asymptomatic SARS-CoV-2 infection: a narrative review. Ann Intern Med. 2020;173: 362-7. https://doi.org/10.7326/M20-3012

17. Ayoub HH, Chemaitelly H, Seedat S, Makhoul M, Al Kanaani Z, Al Khal A, et al. Mathematical modeling of the SARS-CoV-2 epidemic in Qatar and its impact on the national response to COVID-19. J Glob Health. 2021;11:05005. https://doi.org/10.7189/jogh.11.05005

18. Abu-Raddad LJ, Chemaitelly H, Malek JA, Ahmed AA, Mohamoud YA, Younuskunju S, et al. Assessment of the risk of SARS-CoV-2 reinfection in an intense re-exposure setting. Clin Infect Dis. 2020 Dec 14:[Epub ahead of print]. https://doi.org/10.1093/cid/ciaa1846

19. World Health Organization. Population-based age-stratified seroepidemiological investigation protocol for COVID-19 virus infection [cited 2020 Apr 15]. https:/ / apps.who.int/ iris/handle/10665/331656

20. World Health Organization. Clinical management of COVID-19 [cited 2020 May 31]. https:/ / www.who.int/ publications-detail/clinical-management-of-covid-19

21. Muench P, Jochum S, Wenderoth V, Ofenloch-Haehnle B, Hombach M, Strobl M, et al. Development and validation of the Elecsys Anti-SARS-CoV-2 immunoassay as a highly specific tool for determining past exposure to SARS-CoV-2. J Clin Microbiol. 2020;58:e01694-20. https:/ / doi.org/10.1128/ JCM.01694-20

22. The Roche Group. Roche's COVID-19 antibody test receives FDA emergency use authorization and is available in markets accepting the CE mark [cited 2020 Jun 5]. https:/ / www.roche.com/media/releases/med-cor-202005-03.htm

23. Thermo Fisher Scientific. TaqPath ${ }^{\mathrm{TM}}$ COVID-19 CE-IVD RT-PCR Kit instructions for use [cited 2020 Dec 2]. https:/ / assets.thermofisher.com/TFS-Assets/LSG/ manuals/MAN0019215_TaqPathCOVID-19_CE-IVD_ RT-PCR\%20Kit_IFU.pdf

24. Kubina R, Dziedzic A. Molecular and serological tests for COVID-19 a comparative review of SARS-CoV-2 coronavirus laboratory and point-of-care diagnostics. Diagnostics (Basel). 2020;10:E434. https://doi.org/10.3390/diagnostics10060434

25. US Food and Drug Administration. Cobas $₫$ SARS-CoV-2: Qualitative assay for use on the cobas ${ }^{\circledR} 6800 / 8800$ systems [cited 2020 Dec 2]. https:/ / www.fda.gov/media/136049/ download

26. DerSimonian R, Laird N. Meta-analysis in clinical trials. Control Clin Trials. 1986;7:177-88. https://doi.org/10.1016/ 0197-2456(86)90046-2

27. Miller JJ. The inverse of the Freeman: Tukey double arcsine transformation. Am Stat. 1978;32:138 [cited 2020 Dec 2]. https://www.tandfonline.com/doi/abs/10.1080/00031305.1 978.10479283

28. Barendregt JJ, Doi SA, Lee YY, Norman RE, Vos T. Meta-analysis of prevalence. J Epidemiol Community Health. 2013;67:974-8. https://doi.org/10.1136/jech-2013-203104

29. StataCorp. Statistical Software: Release 16.1. College Station (TX): Stata Corporation; 2019.

30. MATLAB®. The language of technical computing. Natick (MA): The MathWorks, Inc.; 2019.

31. Sethuraman N, Jeremiah SS, Ryo A. Interpreting diagnostic tests for SARS-CoV-2. JAMA. 2020;323:2249-51.

https://doi.org/10.1001/jama.2020.8259

32. Wajnberg A, Mansour M, Leven E, Bouvier NM, Patel G, Firpo-Betancourt A, et al. Humoral response and PCR positivity in patients with COVID-19 in the New York City region, USA: an observational study. Lancet Microbe. 2020; 1:e283-9. https:/ / doi.org/10.1016/S2666-5247(20)30120-8

33. Dan JM, Mateus J, Kato Y, Hastie KM, Yu ED, Faliti CE, et al. Immunological memory to SARS-CoV-2 assessed for up to 8 months after infection. Science. 2021;371:eabf4063. https://doi.org/10.1126/science.abf4063

34. Wajnberg A, Amanat F, Firpo A, Altman DR, Bailey MJ, Mansour M, et al. Robust neutralizing antibodies to SARS-CoV-2 infection persist for months. Science. 2020;370:1227-30. https://doi.org/10.1126/science.abd7728

35. Lumley SF, O'Donnell D, Stoesser NE, Matthews PC, Howarth A, Hatch SB, et al.; Oxford University Hospitals Staff Testing Group. Antibody status and incidence of SARS-CoV-2 infection in health care workers. N Engl J Med. 2021;384:533-40. https://doi.org/10.1056/ NEJMoa2034545

36. Anderson RM, Heesterbeek H, Klinkenberg D, Hollingsworth TD. How will country-based mitigation measures influence the course of the COVID-19 epidemic? Lancet. 2020;395:931-4. https:// doi.org/10.1016/ S0140-6736(20)30567-5

37. Britton T, Ball F, Trapman P. A mathematical model reveals the influence of population heterogeneity on herd immunity to SARS-CoV-2. Science. 2020;369:846-9. https://doi.org/10.1126/science.abc6810 
38. He W, Yi GY, Zhu Y. Estimation of the basic reproduction number, average incubation time, asymptomatic infection rate, and case fatality rate for COVID-19: Meta-analysis and sensitivity analysis. J Med Virol. 2020;92:2543-50. https:/ / doi.org/10.1002/jmv.26041

39. Online MIDAS. COVID-19 portal. COVID-19 parameter estimates: basic reproduction number [cited 2020 May 19] https://github.com/midas-network/COVID-19/tree/ master/parameter_estimates/2019_novel_coronavirus

40. Public Health England. Evaluation of Roche Elecsys AntiSARS-CoV-2 serology assay for the detection of anti-SARS-CoV-2 antibodies [cited 2020 Jun 5]. https://assets.publishing.service.gov.uk/government/ uploads/system/uploads/attachment_data/file/891598/ Evaluation_of_Roche_Elecsys_anti_SARS_CoV_2 PHE_200610_v8.1_FINAL.pdf

41. Oved K, Olmer L, Shemer-Avni Y, Wolf T, Supino-Rosin L, Prajgrod G, et al. Multi-center nationwide comparison of seven serology assays reveals a SARS-CoV-2 non-responding seronegative subpopulation. EClinicalMedicine. 2020; 29:100651. https://doi.org/10.1016/j.eclinm.2020.100651

42. Pollán M, Pérez-Gómez B, Pastor-Barriuso R, Oteo J, Hernán MA, Pérez-Olmeda M, et al.; ENE-COVID Study Group. Prevalence of SARS-CoV-2 in Spain (ENE-COVID): a nationwide, population-based seroepidemiological study. Lancet. 2020;396:535-44. https:// doi.org/10.1016/ S0140-6736(20)31483-5

43. Makhoul M, Ayoub HH, Chemaitelly H, Seedat S, Mumtaz GR, Al-Omari S, et al. Epidemiological impact of
SARS-CoV-2 vaccination: mathematical modeling analyses. Vaccines (Basel). 2020;8:E668. https:/ / doi.org/10.3390/ vaccines 8040668

44. Makhoul M, Chemaitelly $\mathrm{H}$, Ayoub HH, Seedat $\mathrm{S}$, Abu-Raddad LJ. Epidemiological differences in the impact of COVID-19 vaccination in the United States and China. Vaccines (Basel). 2021;9:223-36. https:/ / doi.org/10.3390/ vaccines 9030223.

45. Jackson LA, Anderson EJ, Rouphael NG, Roberts PC, Makhene M, Coler RN, et al.; mRNA-1273 Study Group. An mRNA vaccine against SARS-CoV-2: preliminary report. N Engl J Med. 2020;383:1920-31. https:/ /doi.org/10.1056/ NEJMoa2022483

46. Polack FP, Thomas SJ, Kitchin N, Absalon J, Gurtman A, Lockhart S, et al.; C4591001 Clinical Trial Group. Safety and efficacy of the BNT162b2 mRNA COVID-19 vaccine. N Engl J Med. 2020;383:2603-15. https:/ / doi.org/10.1056/ NEJMoa2034577

Address for correspondence: Andrew Jeremijenko, Hamad Medical Corporation, PO Box 3050, Doha, Qatar; email: ajeremijenko@hamad.qa or Laith J. Abu-Raddad, Infectious Disease Epidemiology Group, World Health Organization Collaborating Centre for Disease Epidemiology Analytics on HIV/AIDS, Sexually Transmitted Infections, and Viral Hepatitis, Weill Cornell Medicine, Qatar, Qatar Foundation, Education City, PO Box 24144, Doha, Qatar; email: 1ja2002@qatar-med.cornell.edu

\section{EID Podcast:}

\section{Endotheliopathy and Platelet Dysfunction as Hallmarks of Fatal Lassa Fever}

\section{Lassa fever, a virus spread through the inhalation of rodent excreta, often causes mild, influenza-like}

symptoms. But in severe cases, patients can face bleeding, neurological symptoms, and a death rate up to 70 percent.

Lassa fever alters platelet function and blood clotting, but the exact mechanisms involved remain a mystery. Now, researchers are searching for answers.

In this EID podcast, Dr. Brian Sullivan, a researcher and instructor at La Jolla Institute for Immunology, discusses how Lassa fever affects the vascular system. 